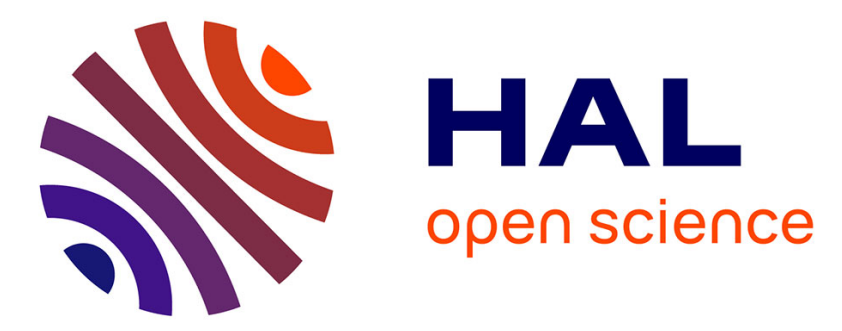

\title{
Surface-induced Frustration in Solid State Polymorphic Transition of Native Cellulose Nanocrystals
}

\author{
Reeta Salminen, Niki Baccile, Mehedi Reza, Eero Kontturi
}

\section{To cite this version:}

Reeta Salminen, Niki Baccile, Mehedi Reza, Eero Kontturi. Surface-induced Frustration in Solid State Polymorphic Transition of Native Cellulose Nanocrystals. Biomacromolecules, 2017, 18 (6), pp.1975-1982. 10.1021/acs.biomac.7b00463 . hal-01518291

\section{HAL Id: hal-01518291 \\ https: / hal.sorbonne-universite.fr/hal-01518291}

Submitted on 4 May 2017

HAL is a multi-disciplinary open access archive for the deposit and dissemination of scientific research documents, whether they are published or not. The documents may come from teaching and research institutions in France or abroad, or from public or private research centers.
L'archive ouverte pluridisciplinaire HAL, est destinée au dépôt et à la diffusion de documents scientifiques de niveau recherche, publiés ou non, émanant des établissements d'enseignement et de recherche français ou étrangers, des laboratoires publics ou privés. 


\title{
Surface-induced Frustration in Solid State
}

\section{Polymorphic Transition of Native Cellulose}

\author{
Nanocrystals
}

\author{
Reeta Salminen, ${ }^{a}$ Niki Baccile, ${ }^{b}$ Mehedi Reza, ${ }^{c}$ Eero Kontturi*a
}

\section{AUTHOR ADDRESS}

a Department of Bioproducts and Biosystems, School of Chemical Engineering, Aalto University, P.O. Box 16300, 00076 Aalto, Finland.

b Chimie de la Matière Condensée de Paris, Sorbonne Universités, 75005, Paris, France

c Department of Applied Physics, P.O. Box 11100, 00076 Aalto, Finland

\begin{abstract}
KEYWORDS: cellulose nanocrystals, host-guest system, image analysis, polymorphic transition, surface immobilization
\end{abstract}

\begin{abstract}
The presence of an interface generally influences crystallization of polymers from melt or from solution. Here, by contrast, we explore the effect of surface immobilization in a direct solid state polymorphic transition on individual cellulose nanocrystals (CNCs), extracted from a plant-based origin. The conversion from native cellulose I to cellulose III crystal occurred via a
\end{abstract}


host-guest inclusion of ethylene diamine inside the crystal. $60 \%$ reduction in $\mathrm{CNC}$ width (height) in atomic force microscopy images suggested that when immobilized on a flat modified silica surface, the stresses caused by the inclusion or the subsequent regeneration resulted in exfoliation, hypothetically between the van der Waals bonded sheets within the crystal. Virtually no changes in dimensions were visible when the polymorphic transition was performed to non-immobilized CNCs in bulk dispersion. With reservations and by acknowledging the obvious dissimilarities, the exfoliation of cellulose crystal sheets can be viewed as analogous to exfoliation of 2D structures like graphene from a van der Waals stacked solid. Here, the detachment is triggered by an inclusion of a guest molecule inside a host cellulose crystal and the stresses caused by the firm attachment of the CNC on a solid substrate, leading to detachment of molecular sheets or stacks of sheets.

\section{INTRODUCTION}

The possibility of atoms and molecules to organize themselves in multiple different crystalline structures, i.e., polymorphism is a common feature of materials with famous cases such as ice, silica and carbon. ${ }^{1-4}$ Nearly all crystalline polymers also bear this property. Polymorphism is important because the conformation and/or packing of the material affects its physical properties such as melting point, conductivity, solubility, or mechanical properties. ${ }^{2,4-8}$ Meanwhile, chemical properties are influenced by crystalline packing which often controls the accessibility and energetic state of the molecules, thereby affecting their chemical reactivity. ${ }^{3,5,9}$ The extent (degree) of crystallinity and the quality of the polymorphs can be tuned in various ways, e.g., by helical inducers for polymers. ${ }^{10-12}$

The presence of an interface can significantly affect the crystalline structure of materials, small molecules $^{13}$ and polymers ${ }^{14,15}$ alike. The impact of geometrical confinement in forms of 
droplets, ${ }^{16,17}$ thin films, ${ }^{15,18-23}$ adsorbed structures, ${ }^{24,25}$ composite phases, ${ }^{26-28}$ and blend patterns $^{14,29,30}$ on polymer crystals specifically have been comprehensively investigated. Altogether, the interfacial effect on polymer crystals has far reaching implications in several fields of applications, including drug delivery, ${ }^{31}$ semiconductors, ${ }^{32}$ and photovoltaics. ${ }^{33}$

From the fundamental perspective of interfacial polymer polymorphs, the most common approach is to study crystallization from solution or melt onto a solid surface or perhaps observe the effect of temperature as post treatment that leads to crystallization. ${ }^{13-15}$ Biopolymers, particularly proteins, have also received fair attention in this respect. ${ }^{34,35}$ Furthermore, efforts to elucidate crystallization and melting of monomolecular polymer layers on atomically smooth surfaces have recently undergone an upsurge because of the popular appeal of 2D substrates like graphene. ${ }^{36,37}$ By contrast, actual polymorphic transitions from one crystalline form to another near an interface are generally not investigated and biopolymers present a particularly tricky experimental case for this because of their complex crystallinity. Such solid-state conversions would, however, offer more room for maneuvering the properties of materials that are based on interfacial structures. In this paper, we demonstrate a surface-induced frustration in a polymorphic transition of cellulose, the polysaccharide responsible for the structural scaffold of all plant cells.

In nature, crystalline cellulose occurs always as $\mathrm{I}_{\alpha}$ and $\mathrm{I}_{\beta}$ polymorphs. There are two ways of practically altering the crystalline structure: (i) dissolution/regeneration or alkaline swelling leading to cellulose II form and (ii) treatment in liquid ammonia or some diamines like ethylenediamine (EDA) resulting in cellulose III crystals. Of these, the transition to cellulose III is a solid-state conversion: ammonia or diamine molecules enter the otherwise impenetrable cellulose crystal in a host-guest style system, where at formation, the chains slip into the arrangement with altered hydrogen bonding patterns (Figure 1). The major difference between 
cellulose III and cellulose I is that in cellulose III the chains are not aligned as flat stacking sheets with van der Waals bonding in between but as conjugated sheets with zig-zagging hydrogen bonding between them. ${ }^{38-40}$ This is caused by the primary alcohol rotating into a gauge-trans position from the trans-gauge position during the introduction of the guest molecule into the crystal $^{39,41-44}$ or - as proposed recently - proton hopping from one hydroxyl group to the other. ${ }^{45}$ When the host molecule is removed by rinsing with methanol or ethanol, the gauge-trans position remains in the resulting cellulose III structure (Figure 1). ${ }^{42,43}$ While cellulose I is the strongest and the most inert structure, the increased susceptibility to degradation of cellulose III has elicited suggestions that the polymorphic transition could be used as a pretreatment step when hydrolyzing cellulose into sugars for biofuel production..$^{9,38,46}$

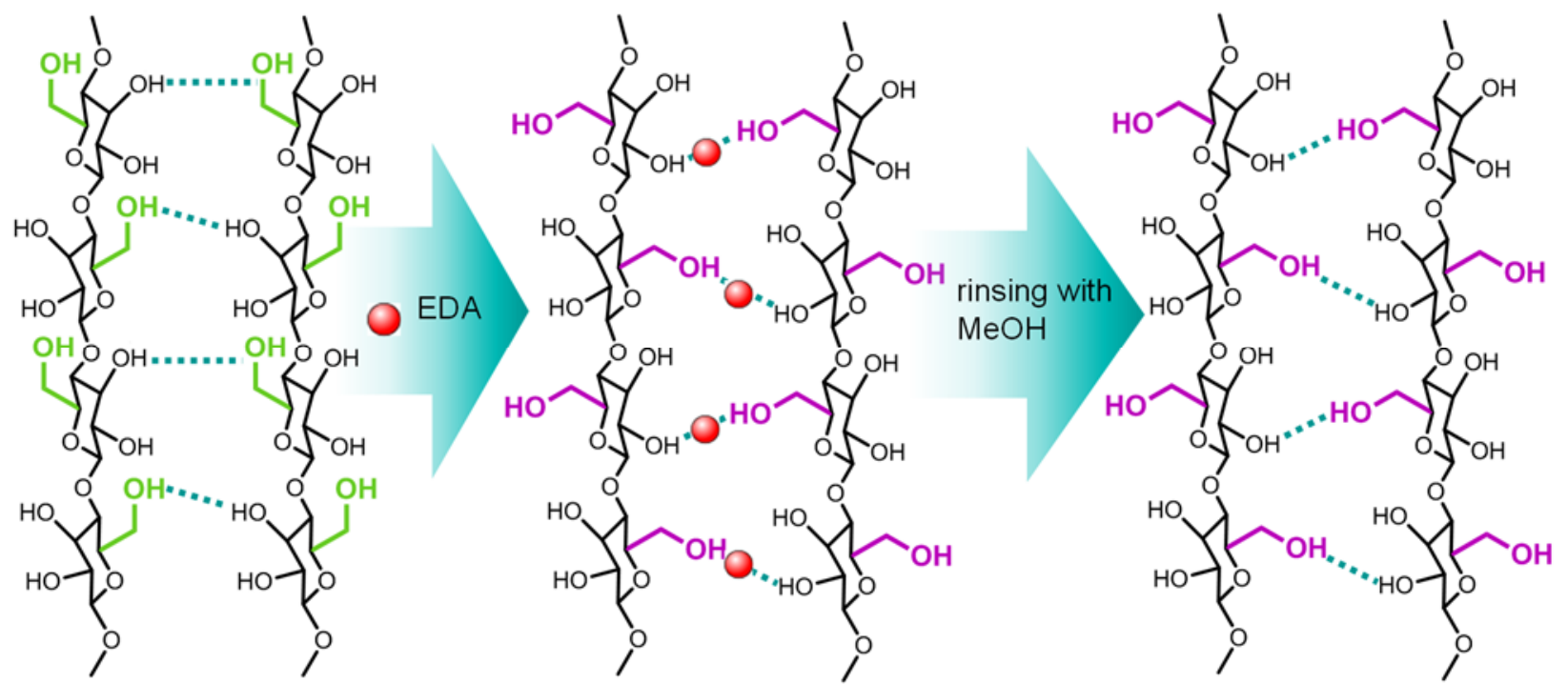

Figure 1. The change in conformation and hydrogen bonding upon EDA-complexation and immersion into methanol.

Here, we utilized the possibility to extract nanosized single crystals of native cellulose I, that is, cellulose nanocrystals (CNCs), directly from plant-based fibers and then closely monitored the morphological changes occurring upon the conversion of cellulose I to cellulose III. This was 
performed with two different methods: a) the polymorphic transition cellulose $\mathrm{I} \rightarrow$ III was done in dispersion (Figure 2a), and b) the CNCs were immobilized on a cationized surface, prior to the $\mathrm{I} \rightarrow \mathrm{III}$ transition (Figure 2b). It turned out that when CNCs were electrostatically immobilized on a flat substrate, the polymorphic transition was visibly frustrated and the alterations could be ascribed to the breakage in the cellulose crystal. More precisely, the van der Waals bonded sheets in the crystal appeared to be exfoliated upon the frustrated polymorphic transition, which can be seen to resemble the exfoliation of 2D sheets like graphene from van der Waals solids. In this particular instance, however, we have a 1D longitudinal crystal and the exfoliation is triggered by a supramolecular entry of a guest molecule (EDA) in a host cellulose crystal instead of physical shear. The results can be seen as the first step in controlling the supramolecular rearrangements of bio-based polymers directly in solid state.

\section{a) Cellulose III CNC, allomorph transformation done in dispersion}

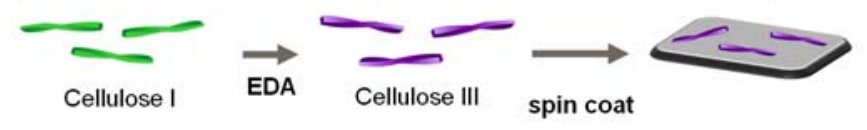

b) Surface immobilized allomorph transition

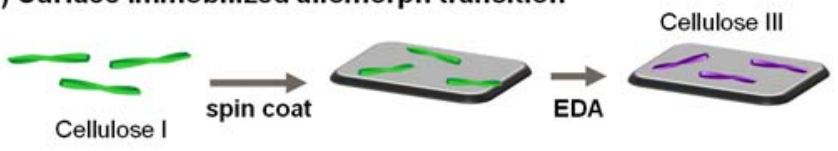

Figure 2. The sample preparation methods: a) The polymorphic transition with ethylenediamine (EDA) is done in dispersion, and then the CNC III is deposited on silicon substrate cationized with 3-aminopropylmethoxysilane (APTS); b) CNC is first deposited on the APTS substrate, and the EDA treatment is performed. 


\section{EXPERIMENTAL}

Materials. Whatman ashless filter paper was purchased from Whatman GmbH, (Dassel, Germany). Sulfuric acid $\left(\mathrm{H}_{2} \mathrm{SO}_{4}, 95-97 \%\right)$ ethylenediamine (EDA, $\geq 99.5 \%$ ), sodium hydroxide $(\mathrm{NaOH})$, toluene (anhydrous, $99.8 \%$ ), methanol ( $\geq 99.8 \%$ ), and (3-aminopropyl)trimethoxysilane (APTS, $97 \%$ ) were purchased from Sigma-Aldrich Finland Oy (Helsinki, Finland). Methanol was dried with $3 \AA$ A molecular sieves (Fluka) also purchased from Sigma-Aldrich Finland Oy (Helsinki, Finland). Ethanol (Aa grade 99.5\% w/v) was purchased from the Altia Corporation (Rajamäki, Finland). Water was purified in a Milli-Q system (Millipore Corporation, resistivity $18.2 \mathrm{M} \Omega \mathrm{cm}$ ). Silicon wafers used as substrates were obtained from Okmetic (Vantaa, Finland), and silicon monoxide coated TEM grids from Science Services GmbH (München, Germany). Nitrogen gas $\left(\mathrm{N}_{2}\right.$, ) was purchased from Oy AGA Ab (Espoo, Finland).

CNC preparation. CNCs were produced from ground Whatman 541 ashless filter paper (15 g) by acid hydrolysis with sulfuric acid $(175 \mathrm{ml}, 64 \mathrm{w}-\%)$ at $45^{\circ} \mathrm{C}$ for $45 \mathrm{~min}$, followed by quenching by addition 31 of MilliQ-water. The CNCs were isolated by centrifugation, followed by purification with dialysis until the conductivity of the dialysis water was $<5 \mu \mathrm{S} .{ }^{47}$ The $\mathrm{CNC}$ counter ion was exchanged to $\mathrm{Na}^{+}$by adjusting the $\mathrm{pH}$ to 7 with $0.1 \mathrm{M} \mathrm{NaOH}$, after which the product was dialysed again and freeze dryed. ${ }^{48}$ The CNCs were further purified by Soxhlet extraction with ethanol in order to remove the surface impurities. ${ }^{49}$

Surface cationization of silicon wafers and TEM grids. The silicon wafers were purified before cationization by immersing them into $3 \mathrm{M} \mathrm{NaOH}$-solution for $20 \mathrm{~s}$, followed by careful and excessive rinsing with MilliQ-water. ${ }^{50}$ The wafers were then dried with $\mathrm{N}_{2}$ and placed into ozonator (Bioforce Nanosciences UV.TC.EU.003) for 15 minutes followed by rinsing with water and drying with $\mathrm{N}_{2}$. The cationization of silicon wafers with APTS was done by immersing the 
cleaned wafers into $1 \%(\mathrm{v} / \mathrm{v})$ APTS toluene solution for 40 minutes, rinsed with approximately $50 \mathrm{ml}$ of toluene and dried in an oven for 30 minutes at $60{ }^{\circ} \mathrm{C} .{ }^{51}$ The TEM grids were cationized with the same method, omitting the purification steps.

Immobilization of CNCs on silicon wafers and TEM grids. The inherently anionic CNCs were then immobilized on the APTS surface (Figure 3 ) by spin coating (4000 rpm, $2200 \mathrm{rpm} \mathrm{s}^{-1}$ ) with WS-650SX-6NPP/LITE spin coater (Laurell Technologies Corporation, North Wales, PA, USA) and were cured in oven for 10 minutes at $80{ }^{\circ} \mathrm{C}$. The low $\mathrm{CNC}$ concentration in the dispersion used $\left(10 \mathrm{mg} \mathrm{dm}^{-3}\right)$ formed submonolayers on the APTS surface. The cellulose III CNC submonolayers were also produced on APTS surfaces, but with a higher dispersion concentration $\left(500 \mathrm{mg} \mathrm{dm}^{-3}\right)$ due to a steep decrease in charge during the polymorphic transition.

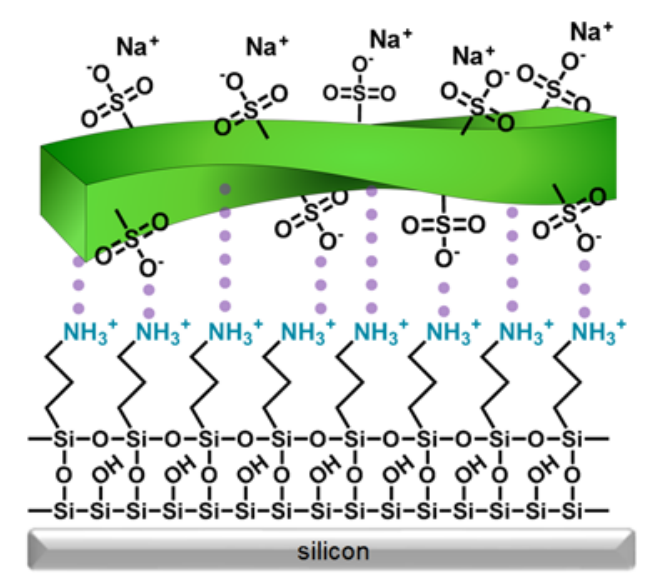

Figure 3. Immobilization of CNC on the cationic APTS surface. The purple dotted lines represent the electrostatic interactions between the cationized surface and the anionic CNC. The scheme is not in scale.

TEM samples were prepared by the CNCs were adsorbed on the cationized TEM grids (10 $\mu 1$, $10 \mathrm{mg} \mathrm{dm}^{-3}$ ), the excess rinsed with milliQ-water, and the excess water blotted carefully with filterpaper. The samples were allowed to air dry before the EDA treatment. For reference samples without the EDA treatment were also imaged. 
The polymorph transition. Cellulose I was transformed into cellulose III by dispersing the CNCs into $75 \%$ aqueous ethylenediamine (EDA) solution for $5 \mathrm{~h}$ at $35^{\circ} \mathrm{C}$, after which the EDA solution was decanted and the CNCs immersed in anhydrous methanol. ${ }^{43}$ The methanol was decanted off and replaced with fresh anhydrous methanol. The washing step was repeated several times. The CNCs were then air dried.

The polymorph transition of immobilized cellulose I to cellulose III were performed on the submonolayers of CNC. In these samples the EDA reaction time was $3 \mathrm{~h}$ in order to protect the presumably delicate cationized surface.

Conductometric titration. Dried particles (0.2 g) were dispersed to $100 \mathrm{ml}$ degassed water and $0.2 \mathrm{ml}$ of $0.5 \mathrm{M} \mathrm{NaCl}$ added. The $\mathrm{CNC}$ was then protonated with $0.1 \mathrm{M} \mathrm{HCl}$, and was stirred for 30 minutes. The dispersion was then titrated with $0.1 \mathrm{M} \mathrm{NaOH}$-solution at rate $0.10 \mathrm{ml} \mathrm{min}^{-1}$ adjusted by 751 GPD Titrino, Metrohm (Herisan, Switzerland). The charge content was determined from the conductivity curve obtained with Metrohm 712 conductometer (Herisan, Switzerland) where only one charged species was detected (sulfate groups). For untreated cellulose I CNCs, the charge was determined as $0.45 \mathrm{mmol} \mathrm{g}^{-1}$ and for the cellulose III CNC, treated with EDA in dispersion, the amount of charged groups was $0.04 \mathrm{mmol} \mathrm{g}^{-1}$.

Atomic Force Microscopy (AFM). The submonolayers were imaged as presented in Figure 2: the cellulose III surfaces as they were and the surface immobilized transition samples before and after the EDA treatment. The imaging was performed with AFM MultiMode 8 scanning probe microscope from Bruker AXS Inc. (Madison, WI, USA) with an E scanner in tapping mode. The cantilevers used were NSC15/AIBS silicon cantilevers from Ultrasharp $\mu$ masch (Tallinn, Estonia). The particle dimensions were obtained using SPIP 6.0.6 particle analysis. The width of the particles was determined from the height scale to overcome the error caused by the AFM tip 
convolution. For sufficient statistical sampling 10 samples of each CNC type was imaged from 4 different spots.

Transmission electron microscopy (TEM). Cellulose I CNC and cellulose III CNC were imaged with a field-emission cryo-Transmission Electron Microscope (cryo-TEM, JEOL JEM3200FSC). The sample temperature was maintained at $-187^{\circ} \mathrm{C}$ during the imaging. The images were taken in bright-field mode using zero-loss energy filtering (Omega type) with a slit width of $20 \mathrm{eV}$. Micrographs were recorded with a Gatan Ultrascan 4000 CCD camera and using Gatan DigitalMicrograph software (Gatan, Pleasanton, CA, USA).

Solid state NMR. ${ }^{13} \mathrm{C}$ solid-state Cross-Polarization (CP) Magic Angle Spinning (MAS) NMR experiments have been acquired on a Bruker Avance $300 \mathrm{MHz}(7 \mathrm{~T})$ spectrometer using $7 \mathrm{~mm}$ zirconia rotor spinning at a MAS frequency of $v_{\mathrm{MAS}}=5 \mathrm{kHz}$. The contact time is set to $2 \mathrm{~ms}$, the recycling delay is set to $5 \mathrm{~s}$ and 320 transients have been used. CP is commonly used for its high sensitivity, however, $\mathrm{CP}$ is also known not to be quantitative as the peak relative intensities depend on the choice of the contact time. For this reason, we have also performed single pulse (SP) experiments using a classical high power heteronuclear decoupling pulse sequence, the disadvantage of SP being the poor signal-to-noise ratio. For these experiments, the recycle delay is set to $60 \mathrm{~s}$ and the number of transients to 120 . Chemical shifts were referenced relative to tetramethylsilane $(\mathrm{TMS} ; \delta=0 \mathrm{ppm})$. Deconvolution is initially performed on the CP-MAS Cellulose I spectrum for its high signal-to-noise ratio and then used to deconvolute the Cellulose III CP-MAS spectrum simply by letting the intensity vary. The SP-MAS spectra are then deconvoluted using the data from CP-MAS and only intensity is allowed to vary.

RESULTS AND DISCUSSION 
The polymorphic transition of CNCs in dispersion (Figure 2a) was followed by solid state nuclear magnetic resonance spectroscopy $\left({ }^{13} \mathrm{C} \mathrm{NMR}\right)$. The conversion from cellulose I to III was performed by first preparing the EDA-cellulose complex from freeze dried CNCs and rinsing the EDA away with methanol - altogether an established procedure for cellulosic fibers ${ }^{43}$ but not previously reported for CNCs. This change to cellulose III in bulk dispersion was determined with ${ }^{13} \mathrm{C}$ MAS NMR both using Cross Polarization (CP) (Figure S3) and single pulse (SP) (Figure 4 and S2), the latter being used as a complement for $\mathrm{CP}$ experiments, more sensitive but known to be not quantitative. Cellulose I and cellulose III can be identified by the difference in chemical shift of the C6 peak, resonating at about 65 ppm and 63 ppm (Table S1), respectively, for cellulose I and cellulose III. ${ }^{39,52,53}$ Before phase change, cellulose I is characterized by two C6 resonances (Figure 4), at $65.66 \mathrm{ppm}$ and $63.04 \mathrm{ppm}$, respectively indicating the bulk and surface contribution of the CNCs, as suggested by Brinkmann et al. ${ }^{54}$ After phase change, the bulk cellulose I contribution at $65.66 \mathrm{ppm}$ is reduced by a factor 2.5 after $\mathrm{CP}$, a value which is confirmed by the quantitative SP experiments (2.3) (Table S1). Meanwhile, the increase in the cellulose III resonance at $63.04 \mathrm{ppm}$ is also estimated to a factor of 2.5. If one neglects, to a first approximation, the surface contribution of cellulose I before and after the phase change, one can reasonably estimate that cellulose III represents, after phase change, an average of $75 \pm 4 \%$ of the material, whereas the respective values for $\mathrm{CP}$ and SP experiments are $71 \%$ and $79 \%$ (see Supporting Information). More than just one EDA treatment would have undoubtedly increased the extent of cellulose III conversion, but we opted for a single treatment because EDA reduced the number of sulfate groups on the $\mathrm{CNC}$ surface and further exposures would have caused more problems in the eventual immobilization of CNCs, based on the attraction between sulfates and the APTS surface. 


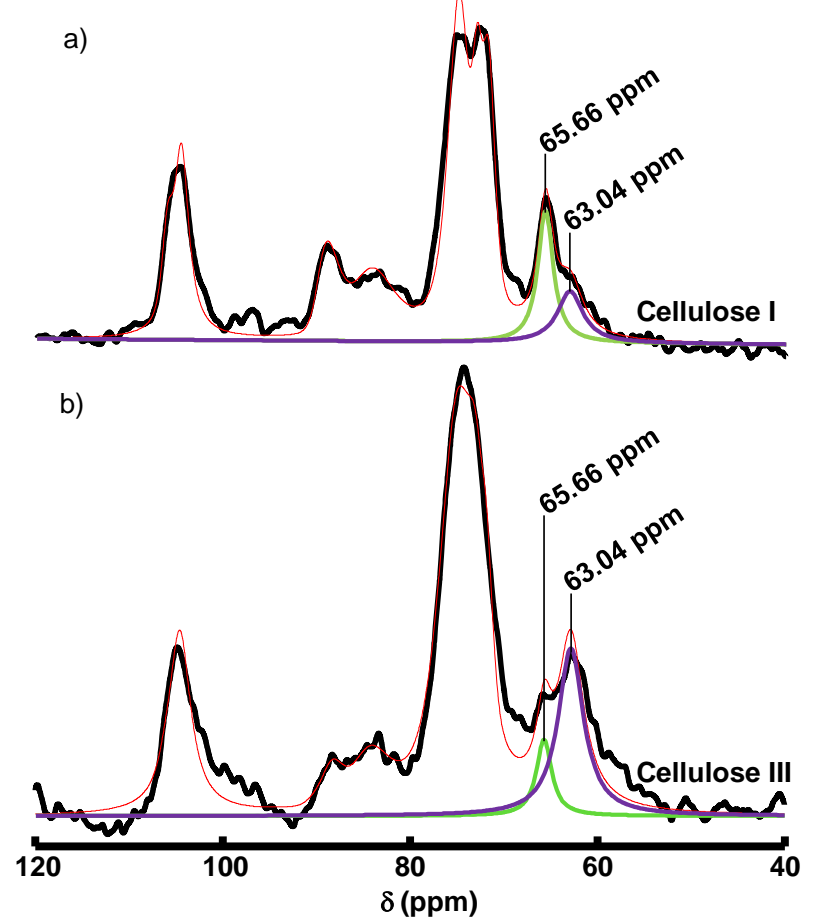

Figure 4. SP-MAS ${ }^{13} \mathrm{C}-\mathrm{NMR}$ spectra of a) cellulose I CNC and b) cellulose III CNC with peak fits for C6-chemical shift. The purple curve is the peak fit to peak $63.04 \mathrm{ppm}$ (cellulose III, and disordered cellulose on the crystal surface) and green to $65.66 \mathrm{ppm}$ (cellulose I). The red curve is the peak fit sum. Full peak fit spectra can be found from Supporting Information (Figure S2).

The morphology of the CNCs upon cellulose I to III conversion in dispersion was followed by AFM (Figure 5) and particle size analysis from the respective images (Figure 6, Figure S1 and Table S2). The CNCs were deposited on a silicon surface that was cationized with 3aminopropylmethoxysilane (APTS). As the CNC surfaces are dotted with anionic sulfate groups, the cationic APTS surface enabled nice separation of the CNCs, allowing a close examination of the crystals individually. However, cellulose III CNCs produced in dispersion required a higher dispersion concentration to yield an examinable submonolayer (Figure S1) because of a decreased surface charge from $0.4 \mathrm{mmol} \mathrm{g}^{-1}$ to $0.04 \mathrm{mmol} \mathrm{g}^{-1}$ as shown by conductometric titration. A similar reduction in surface charge has been observed when CNCs have been treated with alkaline, and 
because EDA is highly alkaline, this was expected. ${ }^{55,56}$ Dimensional analysis from AFM images (Figure 6a) of the original cellulose I CNCs gave average dimensions length of $114 \mathrm{~nm}$ and width of $4.5 \mathrm{~nm}$ (Table S2). CNC widths were actually deduced from CNC height because of the AFM tip deconvolution and because we deemed this as a more appropriate way of analyzing the width of a single cellulose crystal in a CNC. It is generally agreed that most CNCs are actually flat, lateral aggregates of 2-4 crystallites. ${ }^{57}$ When deposited for AFM analysis, the aggregates lie flat on the substrate and the AFM height analysis yields a value for a single crystal in the flat aggregate. ${ }^{57}$ Any compaction of the stiff cellulose crystal upon deposition to a solid substrate is unlikely and has never been reported. Contrary to AFM, TEM allows only the lateral dimensions to be analyzed and the width in the TEM images obviously represents the whole aggregate, ${ }^{57}$ which is also consistent with our TEM results (Figure S4). In conclusion, AFM height analysis from CNC aggregates yields a realistic figure for a single cellulose crystal and this value (4.5 nm, Table S2) is consistent with the values for single crystals in cotton CNCs also in the study that reported the aggregates in the first place. ${ }^{57}$

The width of dispersion method cellulose III crystals in CNCs was very similar to that of the original cellulose I crystals: width $5.2 \mathrm{~nm}$ and length $138.0 \mathrm{~nm}$ (Figure 5b and 6b, Table S2). Transmission Electron Microscopy (TEM) of cellulose I and III CNCs supported the observation that the crystal dimensions remained fairly unchanged before and after the polymorph transition (Figure S4), although the TEM analysis yields the width for the whole aggregate in a CNC. Judging from the unit cell parameters, the crystal dimensions of cellulose I and III should be similar to each other and, therefore, the close resemblance of the CNC dimensions is no surprise. ${ }^{38}$ In contrast, when polymorphic transition was performed by strong aqueous $\mathrm{NaOH}$ solution on individual CNCs to cellulose II crystalline form where the morphology is changed completely from rod-like 
to spherical. ${ }^{55}$ The contrast underlines the fundamental differences between the polymorphic transitions of cellulose I into cellulose II and cellulose III.

When the lattice transition was performed on CNCs immobilized on an APTS treated silicon surface (Figure 2b), the width of the crystals in cellulose III CNCs dropped to half of their original size, $2.1 \mathrm{~nm}$, whereas their average length did not change (Figure 5c and 6c, Table S2). Clearly, some of the CNCs were detached from the surface, possibly due to the decreased electrostatic attraction because of the cleavage of sulfate groups during exposure to EDA since the dissociation of the sulfates should not be affected as EDA is highly alkaline. Therefore, one could question whether the decreased width is just a statistical illusion of thicker crystals being detached and thinner ones remaining on the surface. The number of CNCs on a sampled surface area does indeed decrease from over 2600 (Table S2) to less than 800, but the initial amount of the CNCs thinner than $2 \mathrm{~nm}$ (12 out of 2694) was not enough to explain the thin CNCs (330 out of 726) observed after the EDA treatment as seen from Figure 6. The EDA treatment for the immobilized CNCs was also performed by rinsing with water instead of methanol afterward (Figure 5d), which reportedly does not result in a change in the crystal lattice, i.e., the EDA-cellulose complex reverts back to a somewhat more disordered cellulose I structure. ${ }^{42,43}$ However, the results of water rinsing experiments were that the crystals thinned to an average width of $1.8 \mathrm{~nm}$ (Table S2), i.e., the same as when rinsed with methanol. In conclusion, the crystal thinning with CNCs appears to occur during EDA swelling or regeneration upon its removal and it occurs exclusively to the surface immobilized CNCs because no thinning was observed for the CNCs treated in bulk dispersion (Figures 5 and 6).

Synchrotron-enhanced grazing incidence wide angle x-ray scattering (GI-WAXS) was attempted to corroborate the decrease in crystal width and to elucidate the polymorph transition, 
but the amount of material was too small in the submonolayers to yield a meaningful diffraction pattern.
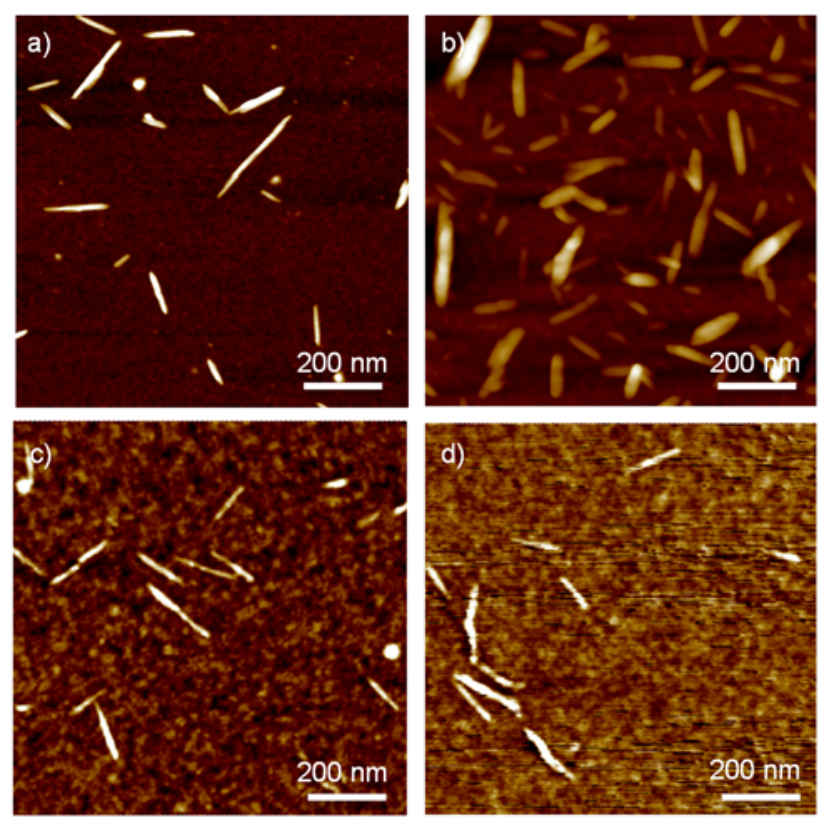

Figure 5. $1 \times 1 \mu \mathrm{m}^{2}$ AFM height images: a) $\mathrm{CNC}$ b) $\mathrm{CNC}$ III, dispersion method c) CNC III, immobilized on the substrate before EDA-treated, rinsed with methanol d) CNC III, immobilized while EDA-treated, rinsed with water. Spherical objects present in a), c) and d) are impurities caused by APTS.
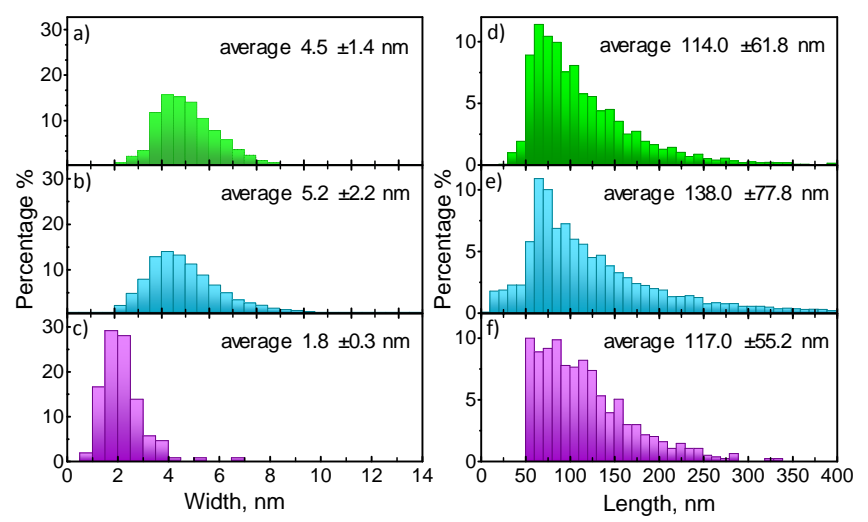
Figure 6. Dimensional distributions as determined from the AFM images. The width distributions are presented on the left column for a) cellulose I CNC, b) cellulose III CNC produced in dispersion, and c) cellulose III CNC produced while immobilized on substrate. The length distributions are on the right side for d) cellulose I CNC, e) cellulose III CNC produced in dispersion, and f) cellulose III CNC produced while immobilized on substrate. The width was determined as the height of CNCs from the substrate surface to omit the tip convolution. The APTS impurities were omitted from the particle analysis.

When the immobilization experiment was repeated with APTS-modified silica-based TEM grids the crystal thinning upon polymorph conversion was not as obvious as with AFM (Figure S5). The APTS-coating on TEM-grids behaved differently than the APTS-coating on silica wafer, i.e., the CNC separation was not as efficient as on a smooth silica surface (Figure S5a). As previously discussed, TEM shows the aggregate size in a $\mathrm{CNC}$ rather than the width of an individual crystallite and this is also evident for both the cellulose III CNCs prepared in dispersion (Figure S5b) and the ones prepared immobilized on the surface (Figure S5b). As a result, overall CNC widths for the cellulose I CNC and cellulose III CNC prepared in dispersion were larger, $15.2 \mathrm{~nm}$ and $18.5 \mathrm{~nm}$ (Figure S5a and S5b), compared to the crystal widths determined by AFM height analysis, $4.5 \mathrm{~nm}$ and $5.2 \mathrm{~nm}$, respectively. Similarly, the EDA treated immobilized CNCs show up as aggregates in TEM images (Figure S5c). However, these CNC aggregates are slightly thinner as $12.9 \mathrm{~nm}$ (Figure $\mathrm{S} 5 \mathrm{c})$ than the reference immobilized cellulose I CNC aggregates (15.2 nm). All in all, although the TEM experiments suggested that CNC thinning upon polymorphic transition does occur, the aggregation impeded making quantitative conclusions on the TEM images and hence the AFM results were regarded as statistically more reliable because the height analysis of the flat aggregates is able to reveal the alterations in the width of an individual crystal in a CNCs. 
As stated before, surface-induced polymer crystallization from solution or from melt is a common phenomenon but an interfacial effect in a solid state polymorphic transition has rarely been explored. The decrease in the CNC width observed particularly with AFM can be hypothetically explained by exfoliation of the outer layers of the crystals during the EDA treatment of the CNCs immobilized on a surface. In the native CNC, i.e., in a cellulose I crystal, the hydrogen bonded cellulose chains form molecularly thin sheets which are stacked on top of each other by van der Waals bonds (Figure 7). ${ }^{58}$ When EDA swells the crystal by host-guest inclusion and the crystal is subjected to stress because of the immobilization, and we can speculate that the weaker van der Waals bonds would be more susceptible to cleavage than the stronger hydrogen bonds which are rearranged in the polymorphic transition process. The flat aggregates of CNCs could be disintegrated as well, but this would not explain the width (height) decrease observed with AFM. To better illustrate the case, we collected several high-resolution AFM images of individual immobilized CNCs that had undergone cellulose I to III conversion (Figure 8). The proposed exfoliation is especially visible in Figures $8 \mathrm{~b}, 8 \mathrm{~d}$ and $8 \mathrm{e}$, where the immobilized CNC appears to have been cleaved in the longitudinal direction, with a thinner crystallite layer still attached to the CNC. Such defibrillation during a polymorphic transition has been previously reported for large cellulose microfibrils in Valonia alga but the authors speculated that the breakage could have been caused by the large size of the crystal, allegedly composed of smaller elementary units. ${ }^{44,52}$ Moreover, the morphological alterations were not induced by the presence of an interface like in our case and the crystal thinning did not appear systematic. It appears that the binding to a surface prior to the host-guest type of entry by EDA into the crystallite causes strain in the lattice (Figure 7). Furthermore, the cellulose chains closest to the cationized surface are hindered from tilting in the cellulose-EDA complex (Figure 7, phases 1 and 2) causing the upper layers to become 
frustrated, and hence prone to break from the lattice and cause thinning. The fact that this phenomenon of exfoliation was also present in the experiments on immobilized CNCs rinsed with water (Figure 5d) strongly suggests that it occurs during the EDA complexation or indeed during the regeneration to either cellulose III or cellulose I upon removal of EDA. In fact, the regeneration can be a more plausible phase to enable exfoliation: the EDA actually plasticizes the swollen crystal while the crystal shrinks during regeneration, rendering it more susceptible to frustration by the underlying interfacial strain. Whatever the mechanisms for the exfoliation may be, the presence of constraints imposed by the surface immobilization are still crucial for the exfoliation to take place since no such phenomenon was observed in cellulose III CNCs produced in a bulk dispersion (Figure 5). 


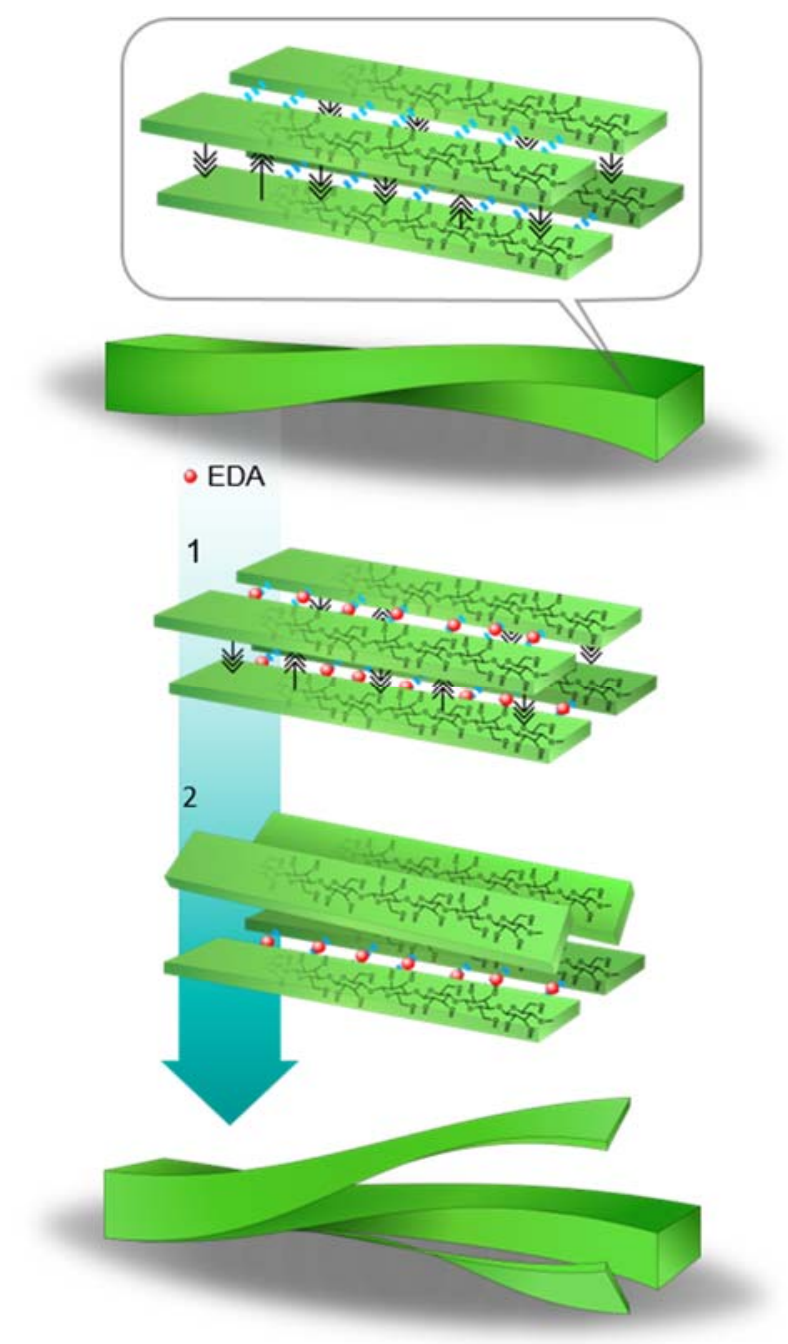

Figure 7. A schematic presentation of cellulose chains in a CNC. When EDA is introduced to for the host-guest complex (1) the lower chains are prevented to tilt (2) hypothetically causing exfoliation of layers of the CNC. 

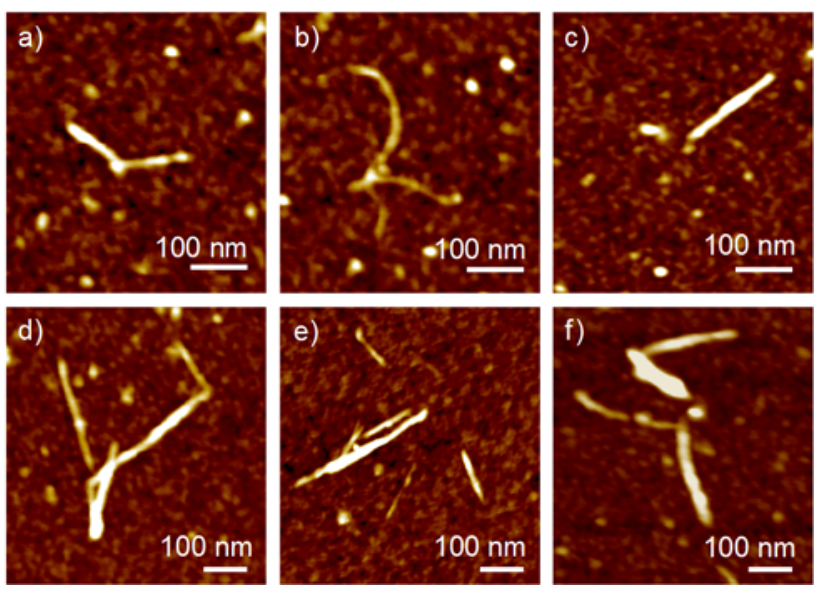

Figure 8. A collection of high resolution AFM height images of broken CNCs caused by EDAcomplex formation while immobilized on APTS-modified substrate. Representative height profiles of these CNCs are presented in the Supporting Information (Figure S6)

In some ways, the structure of the cellulose crystal and the exfoliation of the layers is reminiscent of van der Waals solids and the exfoliation of $2 \mathrm{D}$ structures such as graphene of $\mathrm{MoS}_{2},{ }^{59,60}$ only one of the two lateral dimensions here is just a few molecular layers wide. Indeed, the exfoliation of graphene from graphite is enabled by the individualization of covalently bonded carbon sheets that are van der Waals bonded to one another just like the sheets formed by strong hydrogen bonding in cellulose hypothetically may be detached from one another by EDA inclusion because there is weaker van der Waals bonding between them. ${ }^{61}$ Even covalent breakage of polymer chains by interfacial stress in so-called fatal adsorption has been reported, indicating that the forces can be truly significant upon surface induced frustration. ${ }^{62}$ Furthermore, physical exfoliation of the van der Waals bonded sheets in a cellulose I crystal has been claimed before by a means of high intensity sonication, ${ }^{63}$ further strengthening our hypothesis of selective cleavage of cellulose crystal sheets by EDA. We must emphasize that the attribution of the specific site of exfoliation to the van der Waals bonded sheets is entirely speculative. Further studies in, e.g., molecular 
dynamics simulations are required to further illuminate the case. If the specific site for exfoliation can be verified, this may be useful even in the design of novel cellulose solvents which always require an effective agent to break both the hydrogen bond and the van der Waals bond networks.

\section{CONCLUSION}

When the movement of the CNCs was restricted by electrostatic interactions with the substrate during the polymorphic transition from cellulose I to cellulose III, the average width of individual crystals in CNCs decreased from $4.5 \mathrm{~nm}$ to $2.1 \mathrm{~nm}$ as determined by height analysis from AFM images. However, when transition was performed by EDA treatment to CNCs in dispersion, no changes were observed in the crystal dimensions of CNCs, which was in line with the similar unit cell dimensions of both polymorphs. We hypothesize that the immobilization on the substrate causes frustration in the crystal lattice during the polymorphic transition. The frustration causes the lattice to break, which is observed as a decreased thickness in CNC.

An important note is that the thinning seems to happen during the EDA complex formation or lattice regeneration, since when the immobilized CNCs were rinsed with water after the EDA treatment, the crystal width decreased similarly as when rinsed with methanol. This supports the frustration hypothesis as a reason for the exfoliation, since the frustration would happen only in the presence of the surface-CNC interactions at lattice change either during complexation with EDA or upon regeneration to cellulose I or cellulose III.

In essence, this study reports the chemical exfoliation of molecularly thin structures from a biologically derived crystalline nanomaterial, aided by deliberately built interfacial stress. It may serve as a starting point for similar efforts that can potentially lead to a new family of ultrathin biological materials. 
1
2
3
4
5
6
7
8
9
10
11
12
13
14
15
16
17
18
19
20
21
22
23
24
25
26
27
28
29
30
31
32
33
34
35
36
37
38
39
40
41
42
43
44
45
46
47
48
49
50
51
52
53
54
55
56
57
58
60

ACS Paragon Plus Environment 


\title{
ASSOCIATED CONTENT
}

The following files are available free of charge.

Supporting Information. NMR fitting procedures, TEM images, and further AFM images with height profiles and dimensional data.

\section{AUTHOR INFORMATION}

\section{Corresponding Author}

eero.kontturi@aalto.fi

\section{Funding Sources}

This research is funded by the Academy of Finland (Project 259500).

\section{ACKNOWLEDGMENT}

Dr. Jessie Peyre (UPMC, France) is acknowledged for measuring the charge of untreated CNCs.

\author{
ABBREVIATIONS \\ $\mathrm{CNC}$, cellulose nanocrystal; CP-MAS ${ }^{13} \mathrm{C}-\mathrm{NMR}$, cross-polarization magic angle spin ${ }^{13} \mathrm{C}$-nuclear \\ magnetic resonance; SP-MAS, single pulse magic angel spin; AFM, atomic force microscopy; \\ TEM, transmission electron microscopy; APTS, 3-aminopropyltrimethoxysilane; EDA, \\ ethylenediamine
}




\section{REFERENCES}

1. Gaffney, E. S.; Matson, D. L. Water ice polymorphs and their significance on planetary surfaces. Icarus 1980, 44, 511-519.

2. Keskar, N. R.; Chelikowsky, J. R. Structural properties of nine silica polymorphs. Phys. Rev. B 1992, 46, 1-13.

3. Dubrovinsky, L. S.; Dubrovinskaia, N. A.; Prakapenka, V.; Seifert, F.; Langenhorst, F.; Dmitriev, V.; Weber, H.; Le Bihan, T. A class of new high-pressure silica polymorphs. Phys. Earth Planet. Inter. 2004, 143-144, 231-240.

4. Hazen, R. M.; Downs, R. T.; Jones, A. P.; Kah, L. Carbon Mineralogy and Crystal Chemistry. Rev. Mineral. Geochem. 2013, 75, 7-46.

5. Mercury, L.; Vieillard, P.; Tardy, Y. Thermodynamics of ice polymorphs and 'ice-like' water in hydrates and hydroxides. Appl. Geochem. 2001, 16, 161-181.

6. Tchijov, V. Heat capacity of high-pressure ice polymorphs. Journal of Physics and Chemistry of Solids 2004, 65, 851-854.

7. Li, Q.; Ma, Y.; Oganov, A. R.; Wang, H.; Wang, H.; Xu, Y.; Cui, T.; Mao, H.; Zou, G. Superhard Monoclinic Polymorph of Carbon. Phys. Rev. Lett. 2009, 102, 175506-1-175506-4.

8. Miller, E. D.; Nesting, D. C.; Badding, J. V. Quenchable Transparent Phase of Carbon. Chem. Mater. 1997, 9, 18-22.

9. Igarashi, K.; Wada, M.; Samejima, M. Activation of crystalline cellulose to cellulose IIII results in efficient hydrolysis by cellobiohydrolase. FEBS J. 2007, 274, 1785-1792. 
10. Kawauchi, T.; Kumaki, J.; Kitaura, A.; Okoshi, K.; Kusanagi, H.; Kobayashi, K.; Sugai, T.;

Shinohara, H.; Yashima, E. Encapsulation of Fullerenes in a Helical PMMA Cavity Leading to a Robust Processable Complex with a Macromolecular Helicity Memory. Angew. Chem. 2007, $120,525-529$.

11. Yashima, E.; Matsushima, T.; Okamoto, Y. Poly((4-carboxyphenyl)acetylene) as a Probe for Chirality Assignment of Amines by Circular Dichroism. J. Am. Chem. Soc. 1995, 177, 1159611597.

12. Inai, Y.; Tagawa, K.; Takasu, A.; Hirabayashi, T.; Oshikawa, T.; Yamashita, M. Induction of One-Handed Helical Screw Sense in Achiral Peptide through the Domino Effect Based on Interacting Its N-Terminal Amino Group with Chiral Carboxylic Acid. J. Am. Chem. Soc. 2000, $122,11731-11732$.

13. Jones, A. O. F.; Chattopadhyay, B.; Geerts, Y. H.; Resel, R. Substrate-Induced and ThinFilm Phases: Polymorphism of Organic Materials on Surfaces. Adv. Funct. Mater. 2016, 26, 2233-2255.

14. Michell, R. M.; Müller, A. J. Confined crystallization of polymeric materials. Prog. Polym. Sci. 2016, 54-55, 183-213.

15. Li, H.; Yan, S. Surface-Induced Polymer Crystallization and the Resultant Structures and Morphologies. Macromolecules 2011, 44, 417-428.

16. Carvalho, J. L.; Dainoki-Veress, K. Homogeneous Bulk, Surface, and Edge Nucleation in Crystalline Nanodroplets. Phys. Rev. Lett. 2010, 105, 237801-237804. 
17. Weber, C. H. M.; Chinche, A.; Krausch, G.; Rosenfeldt, S.; Ballauff, M.; Harnau, L.;

Göttker-Schnetmann, I.; Tong, Q.; Mecking, S. Single Lamella Nanoparticles of Polyethylene.

Nano Lett. 2007, 7, 2024-2029.

18. Frank, C. W.; Rao, V.; Despotopoulou, M. M.; Pease, R. F. W.; Hinsberg, W. D.; Miller, R.

D.; Rabolt, J. F. Structure in thin and ultrathin spin-cast polymer films. Science 1996, 273, 912915.

19. Kumaki, J.; Kawauchi, T.; Yashima, E. Two-Dimensional Folded Chain Crystals of a Synthetic Polymer in a Langmuir - Blodgett Film. J. Am. Chem. Soc. 2005, 127, 5788-5789.

20. Ma, Y.; Hu, W.; Reiter, G. Lamellar Crystal Orientations Biased by Crystallization Kinetics in Polymer Thin Films. Macromolecules 2006, 39, 5159-5164.

21. Toolan, D. T. W.; Isakova, A.; Hodgkinson, R.; Reeves-McLaren, N.; Hammond, O.; Edler, K. J.; Briscoe, W. H.; Arnold, T.; Gough, T.; Topham, P. D.; Howse, J. R. Insights into the In fl uence of Solvent Polarity on the Crystallization of Poly(ethylene oxide) Spin-Coated Thin Films via in Situ Grazing Incidence Wide-Angle X - ray Scattering. Macromolecules 2016, 49, 45794586.

22. Aissou, K.; Kwon, W.; Mumtaz, M.; Antoine, S.; Maret, M.; Portale, G.; Fleury, G.; Hadziioannou, G. Archimedean Tilings and Hierarchical Lamellar Morphology Formed by Semicrystalline Miktoarm Star Terpolymer Thin Films. ACS Nano 2016, 10, 4055-4061.

23. Bisbey, R. P.; DeBlase, C. R.; Smith, B. J.; Dichtel, W. R. Two-dimensional Covalent Organic Framework Thin Films Grown in Flow. J. Am. Chem. Soc. 2016, 138, 11433-11436. 
24. Reiter, G.; Sommer, J. Crystallization of Adsorbed Polymer Monolayers. Phys. Rev. Lett. 1998, 80, 3771-3774.

25. Karki, A.; Nguyen, L.; Sharma, B.; Yan, Y.; Chen, W. Unusual Morphologies of Poly(vinyl alcohol) Thin Films Adsorbed on Poly(dimethylsiloxane) Substrates. Langmuir 2016, 32, 31913198.

26. Xu, J.; Chen, T.; Yang, C.; Li, Z.; Mao, Y.; Zeng, B.; Hsiao, B. S. Isothermal Crystallization of Poly( L -lactide) Induced by Graphene Nanosheets and Carbon Nanotubes: A Comparative Study. Macromolecules 2010, 43, 5000-5008.

27. Laird, E. D.; Wang, W.; Cheng, S.; Li, B.; Presser, V.; Dyatkin, B.; Gogotsi, Y.; Li, C. Y. Polymer Single Crystal-Decorated Superhydrophobic Buckypaper with Controlled Wetting and Conductivity. ACS Nano 2012, 6, 1204-1213.

28. Parros-Bujans, F.; Palomino, P.; Fernandez-Alonso, F.; Rudic, S.; Alegria, A.; Colmenero, J.; Encisco, E. Intercalation and Con fi nement of Poly(ethylene oxide) in Porous Carbon Nanoparticles with Controlled Morphologies. Macromolecules 2014, 47, 8729-8737.

29. Prud'homme, R. E. Crystallization and morphology of ultrathin films of homopolymers and polymer blends. Prog. Polym. Sci. 2016, 55-54, 214-231.

30. Tol, R. T.; Mathot, V. B. F.; Reynaers, H.; Groeninckx, G. In Relationship between Phase Morphology, Crystallization, and Semicrystalline Structure in Immiscible Polymer Blends; Harrats, C., Thomas, S. and Groeninckx, G., Eds.; Micro- and nanostructured multiphase polymer blend systems: Phase Morphology and Interfaces; Taylor and Francis: Boca Raton, 2006; pp 391-420. 
31. Kim, B.; Park, S. W.; Hammond, P. T. Hydrogen-Bonding Layer-by-Layer- Assembled Biodegradable Polymeric Micelles as Drug Delivery Vehicles from Surfaces. ACS Nano 2008, 2 , $386-392$.

32. Lei, Y.; Deng, P.; Lin, M.; Zheng, X.; Zhu, F.; Ong, B. S. Enhancing Crystalline Structural Orders of Polymer Semiconductors for Efficient Charge Transport via Polymer-MatrixMediated Molecular Self-Assembly. Adv. Mater. 2016, 28, 6687-6694.

33. Liu, Y.; Zhao, J.; Li, Z.; Mu, C.; Ma, W.; Hu, H.; Jiang, K.; Lin, H.; Ade, H.; Yan, H. Aggregation and morphology control enables multiple cases of high-efficiency polymer solar cells. Nature Commun. 2014, 5, 5293-1-8.

34. Pechkova, E.; Nicolini, C. Protein nanocrystallography: a new approach to structural proteomics. Trends Biotechnol. 2004, 22, 117-122.

35. Chen, C.; Zuckermann, R. N.; DeYreo, J. J. Surface-Directed Assembly of SequenceDefined Synthetic Polymers into Networks of Hexagonally Patterned Nanoribbons with Controlled Functionalities. ACS Nano 2016, 10, 5314-5320.

36. Gulde, M.; Rissanou, A. N.; Harmandaris, V.; Müller, M.; Schäfer, S.; Ropers, C. Dynamics and Structure of Monolayer Polymer Crystallites on Graphene. Nano Lett. 2016, 16, 6994-7000.

37. Gulde, M.; Schweda, S.; Storeck, G.; Maiti, M.; Hak Ki, Y.; Wodtke, A. M.; Schäfer, S.; Ropers, C. Ultrafast low-energy electron diffraction in transmission resolves polymer/ graphene superstructure dynamics. Science 2014, 345, 200-204. 
38. Chundawat, S. P. S.; Bellesia, G.; Uppugundla, N.; da Costa Sousa, L.; Gao, D.; Cheh, A. M.; Agarwal, U. P.; Bianchetti, C. M.; Phillips, G. N. J.; Langan, P.; Balan, V.; Gnanakaran, S.;

Dale, B. E. Restructuring the Crystalline Cellulose Hydrogen Bond Network

Enhances Its Depolymerization Rate. J. Am. Chem. Soc. 2011, 133, 11163-11174.

39. Wada, M.; Chanzy, H.; Nishiyama, Y.; Langan, P. Cellulose IIII Crystal Structure and Hydrogen Bonding by Synchrotron

X-ray and Neutron Fiber Diffraction. Macromolecules 2004, 37, 8548-8555.

40. Parthasarathi, R.; Bellesia, G.; Chundawat, S. P. S.; Dale, B. E.; Langan, P.; Gnanakaran, S. Insights into Hydrogen Bonding and Stacking Interactions in Cellulose. J. Phys. Chem. A 2011, $115,14191-14202$.

41. Wada, M.; Heux, L.; Isogai, A.; Nishiyama, Y.; Chanzy, H.; Sugiyama, J. Improved Structural Data of Cellulose IIII Prepared in Supercritical Ammonia. Macromolecules 2001, 34, 1237-1243.

42. Wada, M.; Kwon, G. J.; Nishiyama, Y. Structure and Thermal Behavior of a Cellulose I-Ethylenediamine Complex. Biomacromolecules 2008, 9, 2898-2904.

43. Lokhande, H. T.; Shukla, S. R.; Chidambareswaran, P. K.; Patil, N. B. Ethylenediamineinduced conversion of cellulose I to cellulose III. J. Polym. Sci. Polym. Lett. Ed. 1977, 15, 97-99.

44. Roche, E.; Chanzy, H. Electron microscopy study of the transformation of cellulose I into cellulose III in Valonia. Int. J. Biol. Macromol. 1981, 3, 1981-206. 
45. Chen, P.; Marianski, M.; Baldauf, C. H-Bond Isomerization in Crystalline Cellulose IIII: Proton Hopping versus Hydroxyl Flip-Flop. ACS Macro Lett. 2016, 5, 50-54.

46. Ono, Y.; Tanaka, R.; Funahashi, R.; Takeuchi, M.; Saito, T.; Isogai, A. SEC-MALLS analysis of ethylenediamine-pretreated native celluloses in $\mathrm{LiCl} / \mathrm{N}, \mathrm{N}$-dimethylacetamide: softwood kraft pulp and highly crystalline bacterial, tunicate, and algal celluloses. Cellulose 2016, 23, 1639-1647.

47. Edgar, C. D.; Gray, D. G. Smooth model cellulose I surfaces from nanocrystal suspensions. Cellulose 2003, 10, 299-306.

48. Beck, S.; Bouchard, J.; Berry, R. Dispersibility in Water of Dried Nanocrystalline Cellulose. Biomacromolecules 2012, 13, 1486-1494.

49. Labet, M.; Thielemans, W. Improving the reproducibility of chemical reactions on the surface of cellulose nanocrystals: ROP of e-caprolactone as a case study. Cellulose 2011, 18, 607-617.

50. Ahola, S.; Salmi, J.; Johansson, L.; Laine, J.; Österberg, M. Model films from native cellulose nanofibrils. Preparation, swelling, and surface interactions. Biomacromolecules 2008, 9, 1273-1282.

51. van Duffel, B.; Verbiest, T.; Van Elshocht, S.; Persoons, A.; De Schryver, F. C.; Schoonheydt, R. A. Fuzzy Assembly and Second Harmonic Generation of Clay/Polymer/Dye Monolayer Films. Langmuir 2001, 17, 1243-1249. 
52. Chanzy, H.; Henrissat, B.; Vincedon, M.; Tanner, S. F.; Belton, P. S. Solid-state 13C-N.M.R. and electron microscopy study on the reversible cellulose $\mathrm{I} \rightarrow \mathrm{III}$ transformation in Valonia . Carbohydr. Res. 1987, 160, 1-11.

53. Isogai, A.; Usuda, M.; Kato, T.; Toshiyuki, U.; Atalla, R. H. Solid-state CP/MAS ${ }^{13}$ C NMR Study of Cellulose Polymorphs. Macromolecules 1989, 22, 3168-3172.

54. Brinkmann, A.; Chen, M.; Couillard, M.; Jakubek, Z. J.; Leng, T.; Johnston, L. J. Correlating Cellulose Nanocrystal Particle Size and Surface Area. Langmuir 2016, 32, 6105-6114.

55. Jin, E.; Guo, J.; Yang, F.; Zhu, Y.; Song, J.; Jin, Y.; Rojas, O. On the polymorphic and morphological changes of cellulose nanocrystals (CNC-I) upon mercerization and conversion to CNC-II. Carbohydr. Polym. 2016, 143, 327-335.

56. Lokanathan, A.; Khan, M. A. U.; Rojas, O.; Laine, J. Cellulose nanocrystal-mediated synthesis of silver nanoparticles: role of sulfate groups in nucleation phenomena. Biomacromolecules 2014, 15, 373-9.

57. Elazzouzi-Harfaoui, S.; Nishiyama, Y.; Putaux, J. -L.; Heux, L.; Dubreuil, F.; Rochas, C. The Shape and Size Distribution of Crystalline Nanoparticles Prepared by Acid Hydrolysis of Native Cellulose. Biomacromolecules 2008, 9, 57-65.

58. Nishiyama, Y.; Sugiyama, J.; Chanzy, H.; Langan, P. Crystal Structure and Hydrogen Bonding System in Cellulose $\mathrm{I}_{\alpha}$ from Synchrotron X-ray and Neutron Fiber Diffraction. J. Am. Chem. Soc. 2003, 125, 14300-14306.

59. Geim, A. K.; Grigorieva, I. V. Van der Waals heterostructures. Nature 2013, 499, 419-425. 
60. Bhimanapati, G. R.; Lin, Z.; Meunier, V.; Jung, Y.; Cha, J.; Das, S.; Xiao, D.; Son, Y.;

Strano, M. S.; Cooper, V. R.; Liang, L.; Louie, S. G.; Ringe, E.; Zhou, W.; Kim, S. S.; Naik, R.

R.; Sumpter, B. G.; Terrones, H.; Xia, F.; Wang, Y.; Zhu, J.; Akinwande, D.; Alem, N.; Schuller,

J. A.; Schaak, R. E.; Terrones, M.; Robinson, J. A. Recent Advances in Two-Dimensional

Materials beyond Graphene. ACS Nano 2015, 9, 11509-11539.

61. Novoselov, K. S.; Geim, A. K.; Morozov, S. V.; Jiang, D.; Zhang, Y.; Dubonos, I. V.; Firsov, A. A. Electric Field Effect in Atomically Thin Carbon Films. Science 2004, 306, 666-669.

62. Lebedeva, N. V.; Sun, F. C.; Lee, H.; Matyjaszewski, K.; Sheiko, S. S. "Fatal Adsorption" of Brushlike Macromolecules: High Sensitivity of C-C Bond Cleavage Rates to Substrate Surface Energy. J. Am. Chem. Soc. 2008, 130, 4228-4229.

63. Li, Q.; Renneckar, S. Supramolecular Structure Characterization of Molecularly Thin Cellulose I Nanoparticles. Biomacromolecules 2011, 12, 650-659. 
Table of Contents Graphics

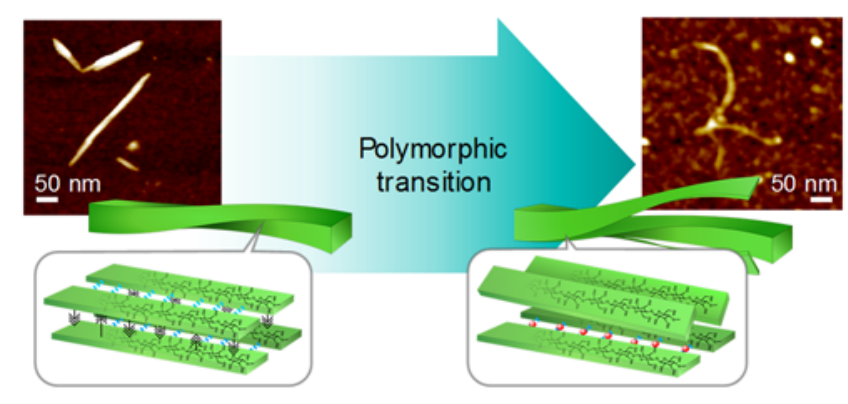

